\title{
Contribuições da monitoria em Fundamentos de Enfermagem II na formação acadêmica de estudantes de enfermagem: relato de experiência
}

\section{Contributions of monitoring in Fundamentals of Nursing II in the academic education of nursing students: experience report}

Contribuciones del seguimiento en Fundamentos de Enfermería II en la formación académica de estudiantes de enfermería: relato de experiencia

Roni Robson da Silva ${ }^{1}$

ORCID: 0000-0001-6010-6438

Carolina da Costa Lipari ${ }^{1}$

ORCID: 0000-0003-2411-2659

Michael Silva Araujo ${ }^{2}$

ORCID: 0000-0002-5389-5712

Leandro Andrade da Silva ${ }^{1}$

ORCID: 0000-0003-3213-5527

Maria Vigirnia Godoy da Silva ${ }^{1}$

ORCID: 0000-0003-3980-042X

Andrezza Serpa Franco ${ }^{1}$

ORCID: 0000-0001-5008-1345

Cristiano Bertolossi Marta ${ }^{1}$

ORCID: 0000-0002-0635-7970

Eliel de Oliveira Larrubia ${ }^{1}$

ORCID: 0000-0003-2994-5991

Marcio Tadeu Ribeiro Francisco ${ }^{1}$

ORCID: 0000-0003-1362-7809

Elson Santos de Oliveira ${ }^{1}$

ORCID: 0000-0001-9377-0140

${ }^{1}$ Universidade do Estado do Rio de Janeiro. Rio de Janeiro, Brasil. ${ }^{2}$ Universidade do Norte do Paraná. Paraná, Brasil.

Como citar este artigo:

Silva RR, Lipari CC, Araujo MS, Silva LA, Silva MVG, Franco AS, Marta CB, Larrubia EO, Francisco MTR, Oliveira ES.

Contribuições da monitoria em Fundamentos de Enfermagem II na formação acadêmica de estudantes de enfermagem: relato de experiência. Glob Acad Nurs. 2021;2(1):e79.

https://dx.doi.org/10.5935/26755602.20200079

Autor correspondente:

Roni Robson da Silva

E-mail: rr.roni1@gmail.com.br

Editor Chefe: Caroliny dos Santos Guimarães da Fonseca

Editor Executivo: Kátia dos Santos Armada de Oliveira

Submissão: 25-10-2020

Aprovação: 20-11-2020

\section{Resumo}

Objetivou-se descrever as contribuições da monitoria de Fundamentos de Enfermagem II na formação acadêmica de estudantes de Enfermagem. Estudo descritivo, tipo relato de experiência, realizado em uma Instituição de Ensino Superior com estudantes de enfermagem na cidade do Rio de Janeiro. Foi possível observar que a monitoria facilitou o aprendizado dos acadêmicos de enfermagem em Fundamentos de Enfermagem II, proporcionou ao acadêmico-monitor a experiência de docência, auxiliou na mediação entre acadêmicos de enfermagem e docente de Fundamentos de Enfermagem II e possibilitou aos acadêmicos a formação de melhor qualidade nesta disciplina como futuros profissionais da enfermagem. Deve-se ressaltar as contribuições da monitoria desta matéria, tanto para os acadêmicos quanto para o acadêmicomonitor, bem como para o docente que ministra esta unidade curricular.

Descritores: Bacharelado em Enfermagem; Docentes de Enfermagem; Educação em Enfermagem.

\section{Abstract}

The aim was to describe the contributions of the Fundamentals of Nursing II monitoring in the academic education of Nursing students. Descriptive study, type of experience report, carried out in a Higher Education Institution with nursing students in the city of Rio de Janeiro. It was possible to observe that the monitoring facilitated the learning of nursing students in Fundamentals of Nursing II, provided the academic monitor with the teaching experience, helped in the mediation between nursing students and a professor of Fundamentals of Nursing II and made it possible for academics to train students. better quality in this discipline as future nursing professionals. It should be emphasized the monitoring contributions of this subject, both for academics and for the academic monitor, as well as for the teacher who teaches this course.

Descriptors: Bachelor of Nursing; Nursing Professors; Nursing Education.

\section{Resumén}

El objetivo fue describir los aportes del seguimiento de Fundamentos de Enfermería II en la formación académica de los estudiantes de Enfermería. Estudio descriptivo, tipo de relato de experiencia, realizado en una Institución de Educación Superior con estudiantes de enfermería de la ciudad de Rio de Janeiro. Se pudo observar que el seguimiento facilitó el aprendizaje de los estudiantes de enfermería en Fundamentos de Enfermería II, brindó al monitor académico la experiencia docente, ayudó en la mediación entre estudiantes de enfermería y un profesor de Fundamentos de Enfermería II y posibilitó que académicos para formar estudiantes de mejor calidad en esta disciplina como futuros profesionales de enfermería. Cabe destacar los aportes de seguimiento de esta asignatura, tanto para los académicos como para el académico-monitor, así como para el docente que imparte esta asignatura.

Descriptores: Licenciatura en Enfermería; Profesores de Enfermería; Educación de Enfermería. 


\section{Introdução}

A graduação em enfermagem objetiva inserir ao estudante competência técnico-científica para o desenvolvimento de atividades assistenciais, gerenciais, de ensino e de pesquisa ${ }^{1}$.

Para maximizar as possibilidades de aprendizagem aos estudantes na execução dos procedimentos de enfermagem, tem-se as atividades acadêmicas complementares desenvolvidas por meio de monitoria acadêmica $^{2,3}$. A monitoria se constitui numa ferramenta relevante no ensino universitário, pela oportunidade de ampliação de experiências que contribuem para sua formação ${ }^{4}$.

O ensino no curso de graduação em enfermagem deve propiciar a aquisição de conhecimentos e mudanças comportamentais, sem perder de vista a vinculação entre teoria e prática ${ }^{1,3,4}$.

A disciplina de Fundamentos de Enfermagem II busca desenvolver no aluno conhecimentos, atitudes e habilidades que o capacitem a prestar assistência de enfermagem ao indivíduo no seu ciclo vital, em seus aspectos técnico-científicos, apoiados na semiologia, no raciocínio clínico, na ética profissional e na sistematização da assistência, em situações que requerem intervenções de enfermagem de menor complexidade ${ }^{5}$. A disciplina é fundamentada nos pressupostos teóricos de Wanda de Aguiar Horta (teoria das necessidades humanas básicas) tendo em vista como uma disciplina complexa por aqueles estudantes que encaram seu estudo de maneira meramente decorada, pois resulta em um processamento de aquisição superficial das informações, e na formação de uma memória menos eficiente ${ }^{4-7}$. A monitoria da Unidade Curricular Fundamentos de Enfermagem II está inserida no curso de Enfermagem no contexto de ensino, extensão e pesquisa7.

$O$ curso de Bacharelado em Enfermagem é reconhecido pela Portaria do MEC n.o 824/014 DOU, de 2 de janeiro de 2015. Na sua matriz curricular, está inserida a unidade curricular Fundamentos de Enfermagem II, oferecida aos discentes do quinto período do curso de graduação, com carga horária teórica e prática de 132 horas, podendo ser monitor de tal unidade apenas os acadêmicos de Enfermagem que já foram aprovados em tal componente, após o quinto período de curso. O estudo de Fundamentos de Enfermagem II está inserido na formação dos acadêmicos de enfermagem, ressaltando não apenas o aprendizado teórico, mas também a prática relacionada à semiologia e semiotécnica, importante para a prestação da assistência à saúde ${ }^{8}$.

Assim, este estudo tem como objetivo descrever as contribuições da monitoria desta disciplina na formação acadêmica de estudantes de Enfermagem de uma universidade na cidade do Rio de Janeiro.

\section{Metodologia}

Estudo descritivo, do tipo relato de experiência, de caráter descritivo, realizado em uma Instituição de Ensino Superior do município do Rio de Janeiro, no período de agosto a dezembro de $2020^{9}$.

$\mathrm{O}$ acadêmico de Enfermagem tornou-se monitor desta disciplina mediante processo seletivo específico, constituído de avaliação teórica e prática. Após avaliação, o monitor selecionado deu início às atividades da monitoria, com a orientação do docente da unidade. As vivências práticas na monitoria dessa matéria foram ofertadas aos discentes do quinto período do curso de Enfermagem, com aproximadamente 60 estudantes de enfermagem, tendo como critério inclusivo de participação estar cursando a referida unidade curricular ${ }^{10}$.

As monitorias de Fundamentos de Enfermagem II realizaram-se por meio de plataformas digitais Skype, WhatsApp, Zoom para preservar o distanciamento social devido a pandemia do COVID-19, foram estabelecidas dez horas semanais de carga horária obrigatória para a sua realização, sendo o acadêmico-monitor da referida unidade curricular o responsável pelo cumprimento desta atividade, com a orientação do docente do curso de enfermagem.

Como critérios para participação nas monitorias, além dos alunos deverem estar cursando a referida unidade curricular, os acadêmicos de enfermagem deveriam ter noventa por cento de presença na disciplina. Além disso, as atividades desenvolvidas baseavam-se na teoria e, principalmente, em vivências práticas em laboratório, com o auxílio do acadêmico monitor, a fim de contemplar o estudo na sua totalidade ${ }^{8,10}$. Por isso, foram utilizados como recursos metodológicos para a realização destas atividades: perguntas dirigidas aos discentes para levantamento de conhecimentos prévios relacionados ao tema; roteiros práticos sobre os assuntos estudados; livros; vivências; realização de simulados teórico, antes das avaliações, para verificação da aprendizagem da unidade curricular; e testes práticos em monitoria ${ }^{11,12}$.

\section{Relato da Experiência}

Durante as vivências práticas na monitoria notou-se o quão importante estas eram tanto para os discentes que participavam com maior frequência quanto para os que participavam com menor frequência ${ }^{9,12}$.

Dentre as contribuições desta atividade na formação acadêmica de estudantes de enfermagem, foi possível observar como resultados: facilitou o processo de ensino aprendizagem dos acadêmicos de enfermagem em Fundamentos de Enfermagem II; proporcionou ao acadêmico-monitor uma experiência de docência ${ }^{7,8,10}$; auxiliou na mediação entre os acadêmicos de enfermagem e o docente, possibilitando aos acadêmicos uma formação de melhor qualidade como futuros profissionais da enfermagem. Neste processo de aprendizado nos cursos superiores, destacam-se o papel do monitor ${ }^{13}$.

A atividade de monitoria tem, em sua essência, a intenção de propiciar ao acadêmico-monitor o início de uma futura carreira docente $\mathrm{f}^{5,15}$. Os acadêmicos apresentaram-se nas monitorias com muitas dificuldades relacionadas ao aprendizado da disciplina ${ }^{5}$. Estes últimos ao participarem da monitoria vivenciavam a experiência de estudar e aprofundar seus conhecimentos, mediante atividades teóricas, onde o aluno tinha a possibilidade de conhecer detalhadamente a elaboração do diagnóstico de enfermagem, semiologia e semiotécnica de enfermagem, 
Contribuições da monitoria em Fundamentos de Enfermagem II na formação acadêmica de estudantes de enfermagem: relato de experiência Silva RR, Lipari CC, Araujo MS, Silva LA, Silva MVG, Franco AS, Marta CB, Larrubia EO, Francisco MTR, Oliveira ES

cálculo de medicação, registro e documentação de Enfermagem ${ }^{3,7,15}$

As relações sociais estabelecidas durante a monitoria, com os professores e colegas, servem de estímulo para o desenvolvimento pessoal do monitor e pode ser um incentivo para o futuro exercício da docência ${ }^{15}$. O acadêmico-monitor exercia o papel de orientar e tirar possíveis dúvidas dos acadêmicos, a fim de construir juntos o conhecimento ${ }^{13,14}$.

Além disso, levando em consideração o nível de complexidade dos assuntos estudados, esta atividade facilitou o processo de ensino-aprendizagem dos acadêmicos de enfermagem em Fundamentos de Enfermagem $\|^{10,12}$. Tendo em vista também a função do acadêmico-monitor de orientar, esclarecer e facilitar o processo de ensino-aprendizagem dos acadêmicos, através da monitoria e de troca da experiência vivenciada anteriormente na unidade, este exerceu uma experiência de docência na sua própria formação acadêmica e auxiliou na mediação entre os acadêmicos de enfermagem e o docente $e^{6,8}$

Esta disciplina apresenta os princípios, os conceitos e as técnicas que constituem os pilares da prática da enfermagem até então desconhecidos por eles e que o guiarão na sua prática profissional ${ }^{2}$. Além disso, todas as contribuições descritas anteriormente proporcionam aos acadêmicos uma formação de melhor qualidade como futuros profissionais da enfermagem, com resultados positivos $^{5,7,10}$.

\section{Conclusão}

Com a realização deste trabalho deve-se ressaltar as contribuições e a importância da monitoria em Fundamentos de Enfermagem II, tanto para os acadêmicos quanto para o acadêmico-monitor, bem como para o docente que ministra esta unidade curricular. A monitoria demanda atitude proatividade do monitor, contribui para a melhoria do ensino, por meio de inovação nas práticas de cuidado em enfermagem, estratégias motivadoras de ensino que possam consolidar as práticas curriculares.

Notou-se o aprimoramento acadêmico diante da oportunidade de vivenciar atividades teóricas e práticas, possibilitando a revisão dos conteúdos e permitindo o aprofundamento nos estudos por meio da atualização constante $\mathrm{e}$ inserção nas atividades em diferentes realidades. Uma vez que na posição de facilitador, seja pela colaboração no planejamento pedagógico e na exposição das aulas, seja pela necessidade de esclarecer dúvidas, superando o individualismo e direcionando as expectativas do outro, isso os leva à busca da ampliação do próprio conhecimento, por meio de sua aprendizagem, resultando em autonomia e responsabilidade na condução do seu projeto de aprendizagem.

A monitoria acadêmica buscou incentivar o desenvolvimento e a análise crítica dos acadêmicos o reflexo dessa ação são discentes mais comprometidos com a qualidade do seu aprendizado. As relações estabelecidas durante a monitoria com os professores e colegas, além de ser um estímulo para o desenvolvimento pessoal do monitor, pode servir de incentivo para o futuro exercício da docência. A monitoria se constitui em uma ferramenta relevante no ensino universitário pela oportunidade de ampliação de experiências que contribuem para a formação acadêmica. Diante dessas possibilidades, este se torna essencial à formação do enfermeiro, devendo as instituições de ensino superior promovê-las e valorizá-las, de forma a somar às competências e habilidades do enfermeiro.

\section{Referências}

1. Guedes MHC, et al. Uso de simulação no ensino de urgência e emergência pré-hospitalar para discentes de medicina: Relato de experiência. Revista de Saúde. 2017;8(1). https://doi.org/10.21727/rs.v8i1.460

2. Lima KRBL, et al. Nursing students experience in emergency and intensive care in a reference hospital. International Archives of medicine. 2016;287(9):1-5. https://doi.org/10.3823/2158

3. Matoso LML. A importância da monitoria na formação acadêmica do monitor: um relato de experiência. Catussaba. 2014;3(2):77-83.

4. Ascari RA, Mahle M. Vivências da monitoria na disciplina de enfermagem no cuidado perioperatório: relato de experiência em 2015.1. In: Universidade do Estado de Santa Catarina. 25 seminário de iniciação científica; 2015.

5. Guedes GF, Cavalcante IMS, Püschel VAA. International academic mobility: the experience of undergraduate nursing students. Rev Esc Enferm USP. 2018;52:e03358. DOI: http://dx.doi.org/10.1590/S1980-220X2017039403358

6. Pinto MB, Medeiros CSA, Andrade LDF de et al. Monitoria Acadêmica: Importância E Contribuição Para A Formação Do Enfermeiro. Rev enferm UFPE on line. 2016;10(6). DOI: 10.5205/reuol.9199-80250-1-SM1006201611

7. Ramos LAV, Costa DS, Cascaes JSA, Souza RTS, Rocha IFC, Galeno NS, Cardoso MM. Plano de monitoria acadêmica na disciplina Anatomia Humana: relato de experiência. Ensino, Saúde e Ambiente. 2012;5(3). https://doi.org/10.22409/resa2012.v5i3.a21015

8. Valsecchi EASS, Nogueira MS. Fundamentos de enfermagem: incidentes críticos relacionados à prestação de assistência em estágio supervisionado. Rev Latino-am Enfermagem. 2002;10(6). https://doi.org/10.1590/s0104-11692002000600011

9. Silva KRX, Oliveira MHS, Souza MM. A monitoria acadêmica como estratégia de enriquecimento curricular. Rev UNIABEU [Internet]. 2016;7(17). Disponível em: https://revista.uniabeu.edu.br/index.php/RU/article/view/1572

10. Nascimento FB, Barletta JB. O olhar do docente sobre a monitoria como instrumento de preparação para a função de professor. Rev Cereus. 2015. https://doi.org/10.1590/0104-07072017002017

11. Dias CLO, Silva Junior RF, Barros SMO. A Monitoria De Educação Em Saúde Na Enfermagem: Relato De Experiência. Rev enferm UFPE on line [Internet]. 2017;11(supl.7):2979-2984. Disponível em: https://pesquisa.bvsalud.org/portal/resource/pt/biblio-1032516

12. Haag GS, et al. Contribuições da monitoria no pr Contribuições da monitoria no processo ensino-aprendizagem em enfermagem. Rev Bras Enferm. 2018;61(2):215-220. https://doi.org/10.1590/S0034-71672008000200011 
Contribuições da monitoria em Fundamentos de Enfermagem II na formação acadêmica de estudantes de enfermagem: relato de experiência Silva RR, Lipari CC, Araujo MS, Silva LA, Silva MVG, Franco AS, Marta CB, Larrubia EO, Francisco MTR, Oliveira ES

13. Freitas CAO, Santos ACM. Uso de metodologias ativas no ensino de práticas de Enfermagem. Rev enferm UFPE on line. 2019;13:e241524 DOI: https://doi.org/10.5205/1981-8963.2019.241524

14. Fernandes NC, et al. Monitoria acadêmica e o cuidado da pessoa com estomia: relato de experiência. Rev Min Enferm. 2015 DOI: $10.5935 / 1415-2762.20150038$

15. Silva RR., et al. Os Impactos do Chemsex na saúde pública mundial: um estudo sobre uma perigosa prática sexual entre homens; Revista Saúde Coletiva Barueri. 2019;51 https://doi.org/10.36489/saudecoletiva.2019v9i51p1920-1925

16. Pereira VFR, et al. Cuidado de enfermagem às pessoas com deficiência na Atenção Primária à Saúde. Glob Acad Nurs 2020;1(1):e7. doi: https://dx.doi.org/10.5935/2675-5602.20200007 